\title{
Investing in Lead-Time Variability Reduction in a Quality-Adjusted Inventory Model with Finite-Range Stochastic Lead-Time
}

\author{
Farrokh Nasri, Javad Paknejad, and John Affisco \\ Department of IT/QM, Frank G. Zarb School of Business, Hofstra University, Hempstead, \\ NY 11549-1340, USA \\ Correspondence should be addressed to Farrokh Nasri, acsfzn@hofstra.edu
}

Received 22 May 2007; Accepted 11 November 2007

Recommended by Ömer S. Benli

\begin{abstract}
We study the impact of the efforts aimed at reducing the lead-time variability in a quality-adjusted stochastic inventory model. We assume that each lot contains a random number of defective units. More specifically, a logarithmic investment function is used that allows investment to be made to reduce lead-time variability. Explicit results for the optimal values of decision variables as well as optimal value of the variance of lead-time are obtained. A series of numerical exercises is presented to demonstrate the use of the models developed in this paper. Initially the lead-time variance reduction model (LTVR) is compared to the quality-adjusted model (QA) for different values of initial lead-time over uniformly distributed lead-time intervals from one to seven weeks. In all cases where investment is warranted, investment in lead-time reduction results in reduced lot sizes, variances, and total inventory costs. Further, both the reduction in lot-size and lead-time variance increase as the lead-time interval increases. Similar results are obtained when lead-time follows a truncated normal distribution. The impact of proportion of defective items was also examined for the uniform case resulting in the finding that the total inventory related costs of investing in lead-time variance reduction decrease significantly as the proportion defective decreases. Finally, the results of sensitivity analysis relating to proportion defective, interest rate, and setup cost show the lead-time variance reduction model to be quite robust and representative of practice.
\end{abstract}

Copyright (C 2008 Farrokh Nasri et al. This is an open access article distributed under the Creative Commons Attribution License, which permits unrestricted use, distribution, and reproduction in any medium, provided the original work is properly cited.

\section{Introduction}

The origin of lot-size research can be traced to the development of the square root EOQ formula in the early 20th century. This relationship is the result of classical optimization of inventoryrelated costs under a series of highly restrictive assumptions. Among these assumptions are instantaneous replenishment, constant deterministic demand and lead-time, and perfect quality of inventory items. More realistic cases ensue when these assumptions are relaxed. Some of 
these cases that have appeared in the literature allow for imperfect quality and variability in either demand or lead-time, or both.

Gross and Soriano [1] and Vinson [2], among others, demonstrate that lead-time variation has a major impact on lot size and inventory costs. Furthermore, they indicate that an inventory system is more sensitive to lead-time variation than to demand variation. The problem of the EOQ model with stochastic lead time has been considered by several additional authors including Liberatore [3], Sphicas [4], and Sphicas and Nasri [5]. In this last work, the authors derive a closed form expression for EOQ with backorders when the range of the leadtime distribution is finite. In this formulation, all units are assumed to be of perfect quality.

Concurrently work has appeared in the literature that relaxes the perfect quality assumption. Rosenblatt and Lee [6] have investigated the effect of process quality on lot size in the classical economic manufacturing quantity model (EMQ). Porteus [7] introduced a modified EMQ model that indicates a significant relationship between quality and lot size. In both $[6,7]$, the optimal lot size is shown to be smaller than that of the EMQ model. In these works, the deterioration of the production system is assumed to follow a random process.

Cheng [8] develops a model that integrates quality considerations with EPQ. (Economic manufacturing quantity.) The author assumes that the unit production cost increases with increases in process capability and quality assurance expenses. Classical optimization results in closed forms for the optimal lot size and acceptable optimal expected fraction. The optimal lot size is intuitively appealing since it indicates an inverse relationship between lot size and process capability.

While this previous work relaxes the perfect quality assumption, it also considers demand to be deterministic. A number of authors have investigated the impact of quality on lot size under conditions of stochastic demand and/or stochastic lead-time. Moinzadeh and Lee [9] have studied the effect of defective items on the operating characteristics of a continuousreview inventory system with Poisson demand and constant lead time. Paknejad et al. [10] present a quality-adjusted lot-sizing model with stochastic demand and constant lead time. Specifically, they investigate the case of continuous-review $(s, Q)$ models in which an order of size $Q$ is placed each time the inventory position (based on nondefective items) reaches the reorder point, $s$. Results indicate that as the probability of defective items increases, for a given constant lead time, the optimal lot size and the optimal reorder point both increase significantly. Further, for a given defective probability, the lot size and the reorder point increase substantially as the lead time increases.

Variations in lead time can occur for purchased items and for those that are manufactured in-house. A major factor related to these variations is quality problems. Typically, either safety stock or safety lead time is utilized to cushion the impact of this variability. In either case, larger variability requires increased inventories. Heard and Plossl [11] portray high leadtime variability as a major reason for a plant's inability to achieve inventory goals and to incur longer average throughput. This suggests that it would be worthwhile to investigate the relationship between quality and lead-time variability, and their impact on lot size and inventory cost. Paknejad et al. [12] began to study this relationship. The authors develop a qualityadjusted model for the case of an inventory model with finite-range stochastic lead times first presented in [5]. This model assumes that each lot contains a random number of defective units all of which are discovered by the purchaser's inspection process and returned to the vendor at the time of the next delivery. The number of defective units in a lot is assumed to follow a binomial distribution. Further, no crossover of orders is allowed. Closed form results are developed 
for a number of decision variables including the optimal quality-adjusted lot size and the optimal total inventory cost. These closed forms are direct functions of the corresponding optimal decision variables developed in [5]. In fact, when quality is perfect, the quality-adjusted model with finite-range stochastic lead time simply reduces to Sphicas and Nasri [5] basic model. In [12], the quality-adjusted optimal lot-size is shown to depend directly on the variance of lead-time in addition to the typical cost and demand parameters, as well as the proportion of defective units in a lot. In this paper, we derive relationships for the case of investment in reducing lead-time variability in a quality-adjusted model with finite-range stochastic lead time. This result points to a new important line of investigation. That is, the analytical determination of the impact of investing to reduce lead-time variance on lot size in a nonperfect quality environment, and ultimately inventory costs. In this paper, we derive these important analytical results, and investigate their robustness through a series of numerical exercises.

\section{Review of basic models and assumptions}

The basic model considered in this paper is the classic EOQ with constant noninterchangeable demand, backorders, and finite-range stochastic lead time, developed by Sphicas and Nasri [5]. Assuming that orders do not cross, the optimal values of the decision variables, $q_{0}$ and $t_{0}$, the resulting optimal lot size, $Q_{0}$, and the optimal expected average cost per unit time, $\operatorname{EAC}_{0}(q, t)$ are given by

$$
\begin{gathered}
q_{0}=\sqrt{\left[\frac{2 K}{D}+(h+p) V\right]\left(\frac{1}{h}+\frac{1}{p}\right)}, \\
t_{0}=\mu-\sqrt{\Omega\left[\frac{2 K}{(h+p) D}+V\right]} \\
Q_{0}=\sqrt{\left[2 D K+V D^{2}(h+p)\right]\left(\frac{1}{h}+\frac{1}{p}\right)}, \\
\operatorname{EAC}_{0}(q, t)=\sqrt{\left[2 D K+V D^{2}(h+p)\right] /\left(\frac{1}{h}+\frac{1}{p}\right)},
\end{gathered}
$$

where

$D=$ demand per unit time (in units),

$K=$ setup cost per setup,

$h=$ holding cost per (nondefective) unit per unit time,

$p=$ backorder cost per (nondefective) unit per unit time,

$\Omega=h / p$,

$V=$ variance of the lead time,

$q=Q / D=$ number of time units of demand satisfied by each order,

$t=$ time differential between placing an order and the start of $\mathrm{q}$ time units that will be satisfied by a given order,

$E A C_{0}(q, t)=$ expected average cost per unit time. 
Note that (2.3) is the stochastic generalization of EOQ when backorders are allowed. Sphicas and Nasri [5] proved that in terms of the parameters of the model, crossover may not occur if and only if $k \geq k_{2}$, where

$$
\begin{gathered}
k=2 K /(h+p) D, \\
k_{2}=(\mu-\alpha)^{2} / \Omega-V, \quad \text { if } \Omega \leq(\mu-\alpha) /(\beta-\mu), \\
k_{2}=\Omega(\mu-\beta)^{2}-V, \quad \text { if } \Omega \geq(\mu-\alpha) /(\beta-\mu),
\end{gathered}
$$

where

$\alpha=$ lower bound of lead-time distribution,

$\beta=$ upper bound of lead-time distribution,

$\mu=$ mean of lead-time distribution.

This formulation assumes that all the units produced by the vendor, in response to the purchaser's order, are nondefective. Paknejad et al. [12] relax this assumption and extend the stochastic generalization of the EOQ model with no crossover of orders by allowing the possibility that each lot may contain a random number of defective units and develop a qualityadjusted model. Specifically, they assume that each lot contains a random number of defective units. Upon arrival, the purchaser inspects the entire lot piece by piece. The purchaser removes the defective units from the lot and returns them to the vendor at the time of next delivery. It is assumed that the vendor picks up the inspection cost incurred by the purchaser. The purchaser's inventory system, however, incurs an extra cost for holding the defective units in stock until the time they are returned to the vendor. They use the following additional notations:

$$
\begin{aligned}
& h=\text { nondefective holding cost per unit per unit time, } \\
& h^{\prime}=\text { defective holding cost per unit per unit time. }
\end{aligned}
$$

Assuming that the number of nondefective units in a lot of size $Q$ can be described by a binomial random variable with parameters $Q$ and $(1-\theta)$, and defining $\rho$, the quality parameter, as the ratio of the probability of a defective unit to the probability of a nondefective unit, that is,

$$
\rho=\frac{\theta}{1-\theta},
$$

and the expected average cost per unit time given that a lot of size $Q$ is ordered, is

$$
\begin{aligned}
& \operatorname{EAC}_{\text {adj }}(q, t) \\
& =\frac{K(1+\rho)}{q}+\frac{D(1+\rho)}{2 q}(h+p)\left[V+(t-\mu)^{2}\right]+D h(t-\mu)+\frac{D h}{2}\left[\frac{\rho+D q}{(1+\rho) D}\right]+h^{\prime} D q-\frac{h^{\prime} D q}{1+\rho} .
\end{aligned}
$$

The optimal values for the decision variables $q_{\mathrm{adj}}^{*} t_{\text {adj }}^{*}$ the resulting optimal lot size $Q_{\text {adj }}^{*}$ and optimal expected average cost per unit time $\operatorname{EAC}_{\text {adj }}^{*}(q, t)$, are found using calculus as follows:

$$
\begin{gathered}
q_{\mathrm{adj}}^{*}=\frac{1+\rho}{\eta} q_{0}, \\
t_{\mathrm{adj}}^{*}=\mu+\frac{t_{0}-\mu}{\eta},
\end{gathered}
$$


Farrokh Nasri et al.

$$
\begin{gathered}
Q_{\mathrm{adj}}^{*}=\frac{1+\rho}{\eta} Q_{0}, \\
\operatorname{EAC}_{\mathrm{adj}}^{*}(q, t)=\frac{h}{2}\left(\frac{\rho}{1+\rho}\right)+\eta \operatorname{EAC}_{0}(q, t),
\end{gathered}
$$

where

$$
\eta=\left[1+2 h^{\prime} \rho\left(\frac{1}{h}+\frac{1}{p}\right)\right]^{1 / 2},
$$

and $q_{0}, t_{0}, Q_{0}$, and $\operatorname{EAC}_{0}(q, t)$ are given in (2.1) through (2.4), respectively. Note that in (2.10) through (2.13), if the quality paramete $\rho=0$, then quality is perfect and the quality-adjusted model with finite-range stochastic lead time simply reduces to Sphicas and Nasri's basic model with no crossover of orders expressed in (2.1) through (2.4).

\section{The optimal lead-time variability model}

The policy variables in (2.9) are $q$ and $t$ for a fixed lead-time variance, $V$. In this paper, as in [13], we assume that the option of investing to reduce $V$ is available. There is now a cost per unit time, $a_{v}(V)$, of changing the lead-time variance to $V$. Thus we consider $V$ to be a decision variable and aim at minimizing the expected average cost per unit time composed of investment to change $V$, ordering, backordering, nondefective holding, and defective holding costs. Specifically, we seek to minimize

$$
\operatorname{EAC}_{\text {adj }}(q, t, V)=i a_{v}(V)+\operatorname{EAC}_{\text {adj }}(q, t),
$$

subject to

$$
0<V \leq V_{0}
$$

where $i$ is the cost of capital $a_{v}(V)$ is a convex and strictly decreasing function of $V$, as defined before, $\operatorname{EAC}_{\text {adj }}(q, t)$ is the sum of inventory related costs given in (2.9), and $V_{0}$ is the original lead-time variance before any investment is made. We use classical optimization techniques to minimize (3.1) over $q, t$, and $V$, ignoring the $0<V \leq V_{0}$ restriction. Of course, if the optimal $V$ obtained in this way does not satisfy restriction (3.2), we should not make any investment, and the results of the quality-adjusted model of the previous section hold. It should be pointed out that it may not always be possible to carry out the minimization. One case where minimization is possible is that of the logarithmic investment function.

\section{The logarithmic investment function case}

This particular function is used in previous research by Porteus [7, 14] and Paknejad et al. [10] dealing with quality improvement as well as setup cost reduction. Paknejad et al. [13] justified its use in the context of lead-time variance reduction based on the idea that lead-time variability reduction should exhibit decreasing marginal return. Further, since high lead-time variability is inevitably related to poor manufacturing, it is conceivable that the steps taken 
to improve the manufacturing process through improved quality and reduced setup time are closely analogous to that of lead-time variability reduction. In this case, lead-time variance declines exponentially as the investment amount $a_{v}$ increases. That is,

$$
a_{v}(V)=\frac{1}{\Gamma} \ln \frac{V_{0}}{V} \quad \text { for } 0<V \leq V_{0},
$$

where $\Gamma$ is the percentage decrease in $V$ per dollar increase in $a_{v}$. Here, our main objective is to minimize $\operatorname{EAC}_{\text {adj }}(q, t, V)$ after substituting (3.3) and (2.9) into (3.1).

Theorem 3.1. If $V_{0}$ and $\Gamma$ are strictly positive and $k \geq k_{2}$, then the following hold:

(a) $\mathrm{EAC}_{\mathrm{adj}}(q, t, V)$ is strictly convex, if and only if

$$
q>\frac{(1+\rho)(h+p)^{2} D^{2} V^{2} \Gamma}{4 i[2 K+(h+p) D V]}
$$

(b) the optimal values of the decision variables are given by

$$
\begin{aligned}
& V_{\mathrm{adj}}^{*}=\min \left\{V_{0}, V_{\mathrm{imp}}\right\}, \\
& q_{\mathrm{adj}}^{* *}=\min \left\{q_{\mathrm{adj}}^{*} q_{\mathrm{imp}}\right\}= \begin{cases}q_{\mathrm{adj}}^{*} & \text { if } V_{\mathrm{imp}} \geq V_{0}, \\
q_{\mathrm{imp}} & \text { if } V_{\mathrm{imp}}<V_{0},\end{cases} \\
& t_{\mathrm{adj}}^{* *}=\max \left\{t_{\mathrm{adj}}^{*}, t_{\mathrm{imp}}\right\}= \begin{cases}t_{\mathrm{adj}}^{*} & \text { if } V_{\mathrm{imp}} \geq V_{0}, \\
t_{\mathrm{imp}} & \text { if } V_{\mathrm{imp}}<V_{0},\end{cases}
\end{aligned}
$$

where $V_{0}=$ the original lead-time variance, $q_{\mathrm{adj}}^{*}$ and $t_{\mathrm{adj}}^{*}$ are given in (2.10) and (2.11),

$$
\begin{aligned}
V_{\text {imp }} & =\frac{2 i}{\Gamma h p D^{2} \eta^{2}}\left[\frac{i}{\Gamma}+\sqrt{\frac{i^{2}}{\Gamma^{2}}+\frac{2 \eta^{2} D K}{(1 / h+1 / p)}}\right] \\
q_{\text {imp }} & =\frac{(1+\rho)(1 / h+1 / p)}{\eta^{2} D}\left[\frac{i}{\Gamma}+\sqrt{\frac{i^{2}}{\Gamma^{2}}+\frac{2 \eta^{2} D K}{(1 / h+1 / p)}}\right] \\
t_{\text {imp }} & =\mu-\frac{\left[i / \Gamma+\sqrt{i^{2} / \Gamma^{2}+2 \eta^{2} D K /(1 / h+1 / p)}\right]}{\eta^{2} D p}
\end{aligned}
$$

(c) the resulting optimal lot size is given by

$$
Q_{\text {adj }}^{* *}=\min \left\{Q_{\text {adj }}^{*}, Q_{\mathrm{imp}}\right\}= \begin{cases}Q_{\mathrm{adj}}^{*} & \text { if } V_{\mathrm{imp}} \geq V_{0}, \\ Q_{\mathrm{imp}} & \text { if } V_{\mathrm{imp}}<V_{0},\end{cases}
$$

where $Q_{\text {adj }}^{*}$ is given by (2.12) and

$$
Q_{\mathrm{imp}}=\frac{(1+\rho)(1 / h+1 / p)}{\eta^{2}}\left[\frac{i}{\Gamma}+\sqrt{\frac{i^{2}}{\Gamma^{2}}+\frac{2 \eta^{2} D K}{(1 / h+1 / p)}}\right] ;
$$

it should be noted that $V_{\mathrm{imp}}, q_{\mathrm{imp}}, t_{\mathrm{imp}}$, and $Q_{\mathrm{imp}}$ do not depend on $V_{0}$; 
Farrokh Nasri et al.

(d) the resulting optimal expected average cost per unit time is given by

$$
\min \left\{\frac{h}{2}\left(\frac{\rho}{1+\rho}\right)+\eta E A C_{0}(q, t), \frac{i}{\Gamma} \ln \frac{V_{0}}{V_{\mathrm{imp}}}+\frac{h}{2}\left(\frac{\rho}{1+\rho}\right)+\eta \sqrt{2 D K \frac{h p}{h+p}+h p D^{2} V_{\mathrm{imp}}}\right\} .
$$

It should be pointed out that when $V_{\mathrm{imp}} \geq V_{0}$, we should not make any investment. In this case, $V_{0}$ will be used in place of $V_{\mathrm{imp}}$, and (3.7), (3.8), and (3.10) will be replaced by the results of quality-adjusted model of Paknejad et al. [12] given in (2.10) through (2.14). Further, when quality is perfect (i. e., $\rho=0$ ), the results of this paper simply reduce to the optimal lead-time variability model of Paknejad et al. [13]. Finally, when $V_{\text {imp }} \geq V_{0}$ and $\rho=0$, the results of this paper reduce to the corresponding results of the basic EOQ with constant noninterchangeable demand, backorders, and finite-range stochastic lead time, developed by Sphicas and Nasri [5].

Proof. (a) $\mathrm{EAC}_{\mathrm{adj}}(q, t, V)$ is strictly convex if all the principal minors of its Hessian determinants are strictly positive. We proceed by producing the principal minors

$$
\begin{aligned}
& \left|H_{11}\right|=\frac{1+\rho}{q^{3}}\left\{2 K+D(h+p)\left[V+(t-\mu)^{2}\right]\right\}>0 \\
& \left|H_{22}\right|=\frac{(h+p) D(1+\rho)^{2}}{q^{4}}[2 K+D(h+p) V]>0 \\
& \left|H_{33}\right|=\frac{(h+p) D(1+\rho)^{2}}{q^{4}}\left[\frac{2 i K}{\Gamma V^{2}}+\frac{i D(h+p)}{\Gamma V}-\frac{(h+p)^{2} D^{2}(1+\rho)}{4 q}\right]>0
\end{aligned}
$$

where $\left|H_{11}\right|$ and $\left|H_{22}\right|$ are strictly positive, and $\left|H_{33}\right|>0$ if and only if the convexity condition of part (a) of Theorem 3.1 holds.

(b) In order to minimize (3.1), it is necessary that

$$
\frac{\partial \mathrm{EAC}_{\mathrm{adj}}(q, t, \rho)}{\partial q}=\frac{\partial \mathrm{EAC}_{\mathrm{adj}}(q, t, \rho)}{\partial t}=\frac{\partial \mathrm{EAC}_{\mathrm{adj}}(q, t, \rho)}{\partial V}=0
$$

The solution to these equations yields $q_{\mathrm{imp}}, t_{\mathrm{imp}}$, and $V_{\mathrm{imp}}$ of part (b) of Theorem 3.1. To prove that the stationary point $\left(q_{\mathrm{imp}}, t_{\mathrm{imp}}, V_{\mathrm{imp}}\right)$ is a relative minimum, it is sufficient to show that it satisfies the convexity condition of part (a). Setting partial derivative of $\operatorname{EAC}_{\text {adj }}(q, t, \rho)$ with respect to $V$ equal to zero and solving, we find that

$$
V_{\text {imp }}=\frac{2 i q_{\text {imp }}}{\Gamma D(1+\rho)(h+p)} .
$$

Substituting (3.14) into the right-hand side of the convexity condition (3.4), after extensive simplification, the convexity condition reduces to

$$
2(1+\rho) K+i q_{\text {imp }}>0 .
$$

Since $\rho, K, i$, and $q_{\text {imp }}$ are all nonnegative, the convexity condition is satisfied at the point $\left(q_{\text {imp }}, t_{\text {imp }}, V_{\text {imp }}\right)$, and part (b) follows.

(c) This part is the direct result of substituting the optimal values of the decision variables into the total cost formula for the two separate cases of $V_{\mathrm{imp}} \geq V_{0}$ and $V_{\mathrm{imp}}<V_{0}$. 


\section{Numerical examples}

Consider an example where the following parameters are known: $D=5200$ units/year, $K=$ $\$ 500 /$ setup, $h=\$ 10 /$ unit/year, $p=\$ 20 /$ unit/year, $h^{\prime}=\$ 5 /$ unit/year, $i=0.10$, and $\Gamma=$ 0.0005. Table 1 presents the results of calculations for the economic order quantity under three scenarios: the EOQ with uniformly distributed lead time (EOQ-SLT) over a five-week interval, the quality-adjusted EOQ (SLT-QA) with uniformly distributed lead time over a five-week interval, and the EOQ including investment in lead-time variance reduction for the case of uniformlyl distributed lead time over a five-week interval (SLT-QA-LTVR). It is interesting to note that SLT-QA is an upper bound for total cost and economic order quantity for the problem. Investment in lead-time variance reduction in this problem results in a 1.48 week reduction in lead-time interval, a 1.30 week reduction in the standard deviation of lead time, and a 0.74 week reduction in mean lead time. Along with this, a 2.74 percent reduction in lot size and a 0.822 percent reduction in total cost are realized.

Table 2 presents the results of additional calculations aimed at determining the impact of initial lead-time variance, $V_{0}$, on the model developed in this paper. Specifically, the qualityadjusted stochastic lead-time models with and without investment in lead-time variance reduction are compared for uniformly distributed lead-time intervals of one through seven weeks. The value of the technical coefficient $\Gamma$ is 0.0005 for the results presented in this table. For the cases in which lead-time interval is 1,2, or 3 weeks, it can be observed from Table 2 that the optimal value of the variance, $V_{\mathrm{imp}}$, is not less than the original variance, $V_{0}$. (Please note that for ease of display, values for $V_{0}$ and $V_{\text {imp }}$ are presented once for each lead-time interval value.) For these cases, as indicated in (2.12), investment in lead-time variance reduction is not warranted and the optimal lot size value remains the optimal value for the quality-adjusted model while the optimal variance is identical to the initial variance. For the cases where the lead-time interval is 4 through 7 weeks, investment in lead-time variability reduction is worthwhile since $V_{\text {imp }}<V_{0}$. For all these cases, the SLT-QA-LTVR model exhibits reduced lot sizes, variance, and total costs when compared to the SLT-QA model. Both reduction in lot size and lead-time variance increase as the lead-time interval, and thus, initial lead-time variability increases. A collateral result induced by the use of the uniform distribution is that the reduction in leadtime variance is accompanied by a reduction in mean lead-time. This reduction also increases as the lead-time variability increases.

In Table 3, we investigate the impact of investing a greater amount in lead-time variance reduction. This is accomplished by increasing $\Gamma$ to 0.005 from 0.0005 . In this situation, only for the case where lead-time interval is one week, the optimal value of the variance, $V_{\text {imp }}$, is greater than the original variance, $V_{0}$, and hence investment in lead-time variance reduction is not warranted. For all the remaining cases, investment in lead-time variability reduction is worthwhile since $V_{\mathrm{imp}}<V_{0}$. Once again, for all these cases, the SLT-QA-LTVR model exhibits reduced lot sizes, variance, and total costs when compared to the SLT-QA model. Also, both the reduction in lot size and lead-time variance increase as the lead-time interval, and thus, initial lead-time variability increases.

Comparing Tables 2 and 3 results shows that the increase in technical coefficient, $\Gamma$, from 0.0005 to 0.005 results in an increase in lead-time variance reduction, when the lead-time interval is 7 weeks, from 74.53 to 97.52 percent (3.049 to 3.983 weeks ${ }^{2}$ ). Interestingly, this increased lead-time variance reduction is accompanied by an increased reduction in lot size from 7.39 to 9.78 percent ( 77.36 units to 102.3 units). Interestingly, the total cost also decreases from $\$ 7,382.75$ to $\$ 6,999.18$, a reduction of $5.2 \%$. This indicates that the decrease in lead-time variance 
Table 1: Comparative results for a uniform numerical example.

\begin{tabular}{lccc}
\hline Variable & EOQ-SLT & SLT-QA & SLT-QA-LTVR \\
\hline$Q$ (units) & 934.75 & 996.44 & 969.14 \\
$\theta$ & - & 0.2 & 0.2 \\
$V(\mathrm{yr})$ & - & - & 0.0003823 \\
$\mu(\mathrm{yr})$ & - & - & 0.033866 \\
TC $(\$)$ & $6,231.64$ & $7,308.25$ & - \\
$\%$ TC Savings over SLT-QA & - & - & - \\
\hline$K=500, D=5200, h=10, p=20, h^{\prime}=5, \mu_{0}=0.048077, V_{0}=0.0007705, i=0.1, \Gamma=0.0005$. &
\end{tabular}

Table 2: Optimal value for various uniform lead-time variabilities with $\Gamma=0.0005$.

\begin{tabular}{|c|c|c|c|c|c|c|c|c|c|}
\hline & & riable & $\begin{array}{l}Q \\
\text { (units) }\end{array}$ & $V_{0}$ & $V_{\text {imp }}$ & $\mu_{0}$ & $\mu^{*}$ & $\mathrm{TC}(\$)$ & $\begin{array}{l}\text { \%TC Savings } \\
\text { over SLT-QA }\end{array}$ \\
\hline \multirow{14}{*}{$\begin{array}{l}\text { Lead- } \\
\text { time } \\
\text { interval } \\
\text { (weeks) }\end{array}$} & \multirow{3}{*}{1} & QA & 943.73 & & & & & $6,291.68$ & - \\
\hline & & LTVR & 943.73 & 0.00003082 & 0.00038230 & 0.00961538 & 0.00961538 & $6,291.68$ & 0 \\
\hline & & QA & 950.48 & & & & & $6,970.17$ & 一 \\
\hline & \multirow[t]{2}{*}{2} & LTVR & 950.48 & 0.00012327 & 0.00038230 & 0.01923077 & 0.01923077 & $6,970.17$ & 0 \\
\hline & & QA & 961.62 & & & & & $7,052.89$ & - \\
\hline & \multirow[t]{2}{*}{3} & LTVR & 961.62 & 0.00027737 & 0.00038230 & 0.02884615 & 0.02884615 & $7,052.89$ & 0 \\
\hline & & QA & 977.01 & & & & & $7,165.73$ & - \\
\hline & \multirow[t]{2}{*}{4} & LTVR & 969.14 & 0.00049310 & 0.00038230 & 0.03846154 & 0.03386602 & $7,158.90$ & 0.095 \\
\hline & & QA & 996.44 & & & & & $7,308.25$ & - \\
\hline & \multirow[t]{2}{*}{5} & LTVR & 969.14 & 0.00077046 & 0.00038230 & 0.04807692 & 0.03386602 & $7,248.16$ & 0.822 \\
\hline & & QA & $1,019.66$ & & & & & $7,478.51$ & - \\
\hline & \multirow[t]{2}{*}{6} & LTVR & 969.14 & 0.00110947 & 0.00038230 & 0.05769231 & 0.03386602 & $7,321.09$ & 2.105 \\
\hline & & QA & $1,046.50$ & & & & & $7,675.31$ & - \\
\hline & 7 & LTVR & 969.14 & 0.00151011 & 0.00038230 & 0.06730769 & 0.03386602 & $7,352.75$ & 3.812 \\
\hline
\end{tabular}

$K=500, D=5200, h=10, p=20, h^{\prime}=5, i=0.1, \ominus=0.2, \Gamma=0.0005$.

Table 3: Optimal value for various uniform lead-time variabilities with $\Gamma=0.005$.

\begin{tabular}{|c|c|c|c|c|c|c|c|c|c|}
\hline & & riable & $\begin{array}{l}Q \\
\text { (units) }\end{array}$ & $V_{0}$ & $V_{\mathrm{imp}}$ & $\mu_{0}$ & $\mu^{*}$ & $\mathrm{TC}(\$)$ & $\begin{array}{l}\text { \%TC Savings } \\
\text { over SLT-QA }\end{array}$ \\
\hline \multirow{14}{*}{$\begin{array}{l}\text { Lead- } \\
\text { time } \\
\text { interval } \\
\text { (weeks) }\end{array}$} & \multirow{3}{*}{1} & QA & 943.73 & & & & & $6,291.68$ & - \\
\hline & & LTVR & 943.73 & 0.00003082 & 0.00003725 & 0.00961538 & 0.00961538 & $6,291.68$ & 0 \\
\hline & & QA & 950.48 & & & & & $6,970.17$ & - \\
\hline & \multirow[t]{2}{*}{2} & LTVR & 944.20 & 0.00012327 & 0.00003725 & 0.01923077 & 0.01057070 & $6,949.07$ & 0.303 \\
\hline & & QA & 961.62 & & & & & $7,052.89$ & - \\
\hline & \multirow[t]{2}{*}{3} & LTVR & 944.20 & 0.00027737 & 0.00003725 & 0.02884615 & 0.01057070 & 6965.29 & 1.242 \\
\hline & & QA & 977.01 & & & & & $7,165.73$ & - \\
\hline & \multirow[t]{2}{*}{4} & LTVR & 944.20 & 0.00049310 & 0.00038230 & 0.03846154 & 0.01057070 & $6,976.80$ & 2.637 \\
\hline & & QA & 996.44 & & & & & $7,308.25$ & - \\
\hline & \multirow[t]{2}{*}{5} & LTVR & 944.20 & 0.00077046 & 0.00003725 & 0.04807692 & 0.01057070 & $6,985.72$ & 4.413 \\
\hline & & QA & $1,019.66$ & & & & & $7,478.51$ & - \\
\hline & \multirow[t]{2}{*}{6} & LTVR & 944.20 & 0.00110947 & 0.00003725 & 0.05769231 & 0.01057070 & $6,993.02$ & 6.492 \\
\hline & & QA & $1,046.50$ & & & & & $7,675.31$ & - \\
\hline & 7 & LTVR & 944.20 & 0.00151011 & 0.00003725 & 0.06730769 & 0.01057070 & $6,999.18$ & 8.809 \\
\hline
\end{tabular}

$K=500, D=5200, h=10, p=20, h^{\prime}=5, i=0.1, \ominus=0.2, \Gamma=0.005$. 
Table 4: Optimal value for various normal lead-time variabilities.

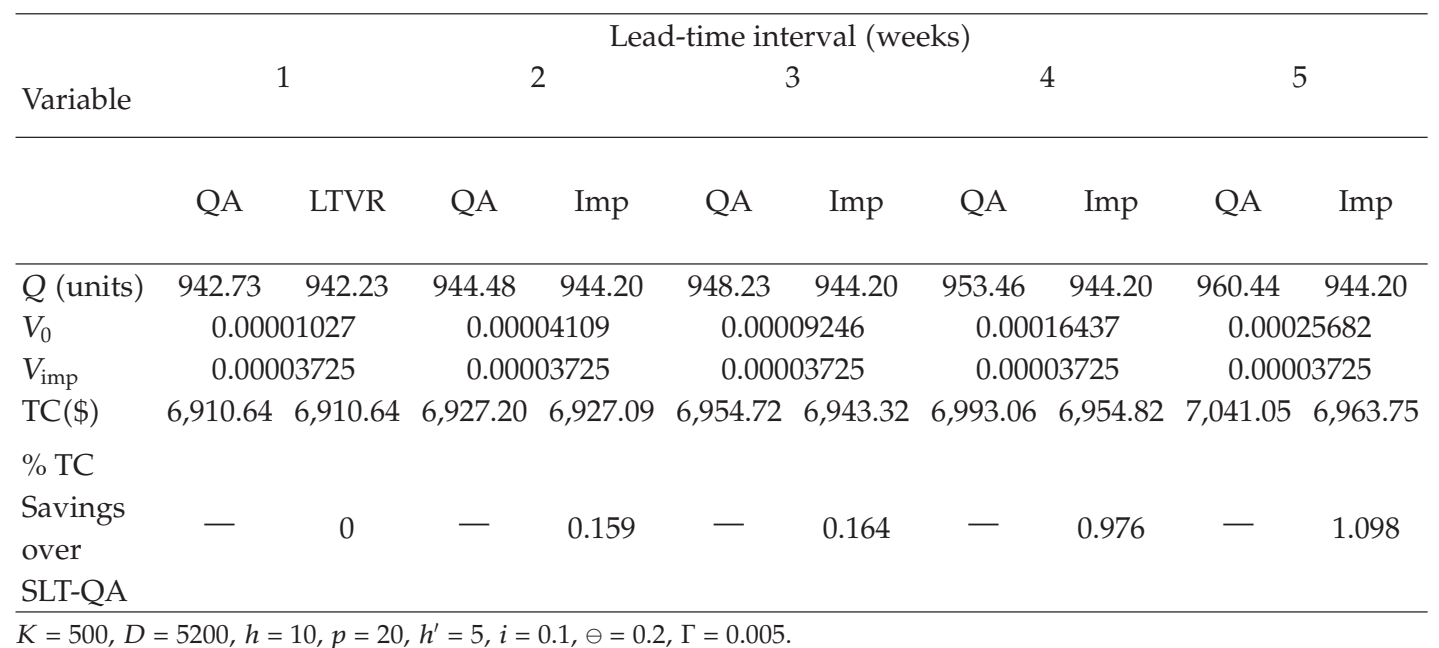

Table 5: Optimal values for various values of $\ominus$.

\begin{tabular}{ccccc}
\hline Variable & $Q$ & $V_{\text {imp }}$ & $\mu^{*}$ & TC (\$) \\
\hline 0.025 & 891.86 & 0.00004288 & 0.01134166 & $6,091.39$ \\
0.050 & 897.93 & 0.00004206 & 0.01123338 & $6,208.25$ \\
0.075 & 904.43 & 0.00004125 & 0.01112462 & $6,330.00$ \\
0.100 & 911.38 & 0.00004045 & 0.01101530 & $6,456.87$ \\
0.125 & 918.79 & 0.00003964 & 0.01090535 & $6,589.08$ \\
0.150 & 926.71 & 0.00003884 & 0.01079466 & $6,726.90$ \\
0.175 & 935.17 & 0.00003804 & 0.01068314 & $6,870.64$ \\
0.200 & 944.20 & 0.00003725 & 0.01057069 & $7,020.65$ \\
0.225 & 953.84 & 0.00003645 & 0.01045722 & $7,177.32$ \\
0.250 & 964.15 & 0.00003566 & 0.01034260 & $7,341.11$ \\
0.275 & 975.17 & 0.00003486 & 0.01022671 & $7,512.52$ \\
0.300 & 986.96 & 0.00003407 & 0.01010941 & $7,692.15$ \\
\hline
\end{tabular}

$K=500, D=5200, h=10, p=20, h^{\prime}=5, i=0.1$, LT Interval $=7$ weeks, $\Gamma=0.005$.

along with the resulting synergistic impact on the lot size clearly improves the overall performance of the production and inventory system.

Table 4 presents similar results, for lead-time intervals from 1 to 5 weeks, when lead time follows a normal distribution which is truncated at $\pm 3 \sigma$. Similar to the uniform case, only for the case where lead-time interval is one week, is the optimal value of the variance, $V_{\text {imp }}$, is greater than the original variance, $V_{0}$, and hence investment in lead-time variance reduction is not warranted. For all the remaining cases, investment in lead-time variability reduction is worthwhile since $V_{\mathrm{imp}}<V_{0}$. Once again, for all these cases, the SLT-QA-LTVR model exhibits reduced lot sizes, variance, and total costs when compared to the SLT-QA model. Also, both the reduction in lot size and lead-time variance increase as the lead-time interval, and thus the initial lead-time variability increases.

For all these results, the proportion defective is 0.20. Equations (3.6) and (3.10) indicate that the optimal lot size and optimal variance for the SLT-QA-LTVR model both depend on 
the proportion defective, $\theta$. Therefore, a logical question to ask is exactly what is the impact of this parameter? Table 5 presents results for defect proportion values from 0.025 to 0.30 for the situation where lead-time follows a uniform distribution with a 7-week lead-time interval and the technical parameter $\Gamma=0.005$. Note that a lead-time variance reduction on the order of $97 \%$ is realized in all cases. There is slightly less of an impact of lead-time variance reduction for a smaller-proportions defective. We also observe a decrease in lot size as the proportion defective decreases. On a relative basis, this decrease is on the order of 5.2 percent when compared to the lot size generated by the solution to the SLT-QA model, increasing slightly on a percentage basis as $\theta$ increases. The total inventory-related cost of implementing SLT-QA-LTVR decreases significantly as the proportion defective decreases. These results provide some preliminary evidence for the concept that programs directed at simultaneously improving quality, and reducing lead-time variability will have a synergistic impact on the performance of production-inventory systems.

\section{Sensitivity analysis}

In this section, we turn our attention to an investigation of the conditions under which investment in lead-time variance reduction is worthwhile. Specifically, we assume both the probabilistic conditions presented in (2.6) and (2.7) and the convexity condition (3.4) are satisfied. Under this scenario, investment is warranted if and only if $V_{\text {imp }}<V_{0}$, which is the equivalent of requiring the optimal lead-time variance to be strictly less than the original lead-time variance, $V_{0}$. By substituting (3.6) for $V_{\text {imp }}$ in this relationship, we may solve for critical points for various parameters of interest in order to perform sensitivity analysis. These derived relationships can provide the manager with a yardstick to determine if investment in lead-time variance reduction would be worthwhile.

Following the procedure outlined above, the critical point for $\rho$ is

$$
\rho>\frac{2 i^{2}}{h^{\prime}(h+p)}\left[\frac{2 K+V_{0} D(h+p)}{(h+p) V_{0}^{2} \Gamma^{2} D^{3}}-h p\right] .
$$

Further, since $\rho=\theta /(1-\theta)$, the critical point for $\theta$ is

$$
\theta>\frac{\rho}{1+\rho} .
$$

Thus when $\theta$ is greater than the right-hand side of (5.2), it pays to invest. Similarly, the critical point for interest rate is

$$
i<\left[\frac{V_{0}^{2} \Gamma^{2} D^{3} \eta^{2} h p}{4\left[2 K /(h+p)+V_{0} D\right]}\right]^{1 / 2} .
$$

Thus when the interest rate is less than the right-hand side of (5.3), it pays to invest. Finally, the critical point for the original setup cost is

$$
K<\frac{(h+p) V_{0} D}{2}\left[\frac{V_{0} \Gamma^{2} D^{2} \eta^{2} h p}{4 i^{2}}-1\right] .
$$


Thus when the setup cost is less than the right-hand side of (5.4), it pays to invest. For each of these relationships, we examine their sensitivity to a single parameter, holding all others constant. We examined a number of cases for which all parameter values are the same as in the sample problem whose results are presented in Table 2. Critical points for proportion defective show the lead-time variability reduction model to be quite robust. As the uniformly distributed lead-time interval increases from two to seven weeks, the lower bound on proportion defective for which the model remains optimal decreases from 0.05 to, essentially, zero. For the following situations, we used a uniformly distributed lead time over a five-week interval. Whether setup cost, $K$, increases from $\$ 250$ to $\$ 2,000$ per setup, annual demand increases from 4000 to 15000 units, or interest rate increases from 2.5 to 20 percent, investment in lead-time reduction is warranted for the smallest possible values of proportion defective. This indicates that even if quality is perfect, there is a benefit to be gained for the performance of the productioninventory system from reducing lead-time variance. Similar results are obtained for variations in the per-unit inventory costs, such as defective holding cost, $h^{\prime}$, nondefective holding cost, $h$, and backorder cost, $p$.

In terms of the critical point for interest rate, as the uniformly distributed lead-time interval increases from one to seven weeks, the upper bound on interest rate for which the model remains optimal increases from 1 to 28.5 percent. As proportion defective, $\theta$, increases from 0.05 to 0.40 , the upper bound on interest rate for which the model remains optimal increases from 14 to 19 percent. In both cases, this is in the correct direction since we would expect to be willing to pay more to reduce lead-time variability when variability is higher or quality is poorer. As the setup cost, $K$, increases from $\$ 250$ to $\$ 2,000$ per setup, the upper bound on interest rate decreases from 29 to 4.3 percent. This makes intuitive sense since high setup cost means large lot sizes and hence a fewer number of replenishment cycles. Thus one would be less willing to pay higher investment costs to reduce lead-time variability when fewer replenishment cycles are experienced by the system. These results suggest that a program of quality improvement should logically be accompanied by one aimed at reducing lead-time variability. The same argument can be made for the case of increasing demand. The results show that as demand increases from 4000 to 15000 units, the upper bound on the interest rate increases from 11.1 to 65.4 percent. Hence, for a given lot size, larger demand induces more replenishment cycles and a willingness to pay more to reduce lead-time variability. Further, improvement in quality will result in smaller lot sizes and, in turn, more replenishment cycles indicating that an accompanying reduction in lead-time variability is in order. An investigation of $\Gamma$ indicates the upper bound on interest rate increases from 1.6 to 32.1 percent as $\Gamma$ increases from 0.000025 to 0.01 indicating that the greater the impact of investment in lead-time variance reduction, the greater the upper bound of what one would be willing to pay to fund such a program. These results indicate that the model is robust with respect to interest rate since, in general, the upper bounds are above the prevailing cost of capital.

Some final interesting results are obtained from an investigation of the critical point for original setup cost, $K_{0}$. As lead-time interval increases from two to seven weeks, the upper bound on original setup cost increases from $\$ 342$ to $\$ 826549$ per setup. This indicates that it is essentially always worthwhile to reduce lead-time variability regardless of the setup cost. Further, the setup cost essentially is no restriction when the lead-time variability is large. When the proportion defective, $\theta$, increases from 0.05 to 0.40 , the upper bound on original setup cost increases from $\$ 168796$ to $\$ 312941$ per setup, indicating that investment in lead-time variability reduction is worthwhile for all cases since actual setup cost for the vast majority of situations 
are lower than the upper bound. These results indicate that there is certainly significant room for reducing setup costs and lead-time variability while simultaneously engaging in a program of quality improvement.

\section{Conclusion}

This paper presents an extension of the quality-adjusted EOQ model with finite-range stochastic lead-times in which investment in lead-time variance reduction is considered. Specifically, a quality-adjusted lead-time variance reduction model is developed in which the lead-time variance is treated as a decision variable. This model assumes investment in lead-time variance reduction proceeds according to a logarithmic investment function. Relationships for economic lot size, optimal total cost, optimal number of time units of demand satisfied by each order, and the optimal time differential between placing an order and the start of $q$ time units that will be satisfied by the given order, as well as optimal lead-time variance, are derived. Results of numerical examples indicate that savings can be realized by investing in lead-time variance reduction. The results of sensitivity analysis relating to proportion defective, interest rate, and setup cost show the lead-time variance reduction model to be quite robust and representative of practice.

\section{References}

[1] D. Gross and A. Soriano, "The effect of reducing lead time on inventory level-simulation analysis," Management Science, vol. 16, pp. B61-B76, 1969.

[2] C. E. Vinson, "The cost of ignoring lead time unreliability in inventory theory," Decision Sciences, vol. 3, no. 2, pp. 87-105, 1972.

[3] M. J. Liberatore, "The EOQ model under stochastic lead time," Operations Research, vol. 27, no. 2, pp. 391-396, 1979.

[4] G. P. Sphicas, "On the solution of an inventory model with variable lead times," Operations Research, vol. 30, no. 2, pp. 404-410, 1982.

[5] G. P. Sphicas and F. Nasri, "An inventory model with finite-range stochastic lead times," Naval Research Logistics Quarterly, vol. 31, no. 4, pp. 609-616, 1984.

[6] M. J. Rosenblatt and H. L. Lee, "Economic production cycles with imperfect production processes," IIE Transactions, vol. 18, no. 1, pp. 48-55, 1986.

[7] E. L. Porteus, "Optimal lot sizing, process quality improvement and setup cost reduction," Operations Research, vol. 34, no. 1, pp. 137-144, 1986.

[8] T. C. E. Cheng, "EPQ with process capability and quality assurance considerations," Journal of Operational Research Society, vol. 42, no. 8, pp. 713-720, 1991.

[9] K. Moinzadeh and H. L. Lee, “A continuous-review inventory model with constant resupply time and defective items," Naval Research Logistics, vol. 34, no. 4, pp. 457-467, 1987.

[10] M. J. Paknejad, F. Nasri, and J. F. Affisco, "Defective units in a continuous review (s,Q) system," International Journal of Production Research, vol. 33, no. 10, pp. 2767-2777, 1995.

[11] E. Heard and G. Plossl, "Lead times revisited," Production and Inventory Management, vol. 23, no. 3, pp. 32-47, 1984.

[12] J. Paknejad, F. Nasri, and J. F. Affisco, “Quality improvement in an inventory model with finite-range stochastic lead times," Journal of Applied Mathematics and Decision Sciences, vol. 2005, no. 3, pp. 177-189, 2005.

[13] M. J. Paknejad, F. Nasri, and J. F. Affisco, "Lead-time variability reduction in stochastic inventory models," European Joural of Operational Research, vol. 62, no. 3, pp. 311-322, 1992.

[14] E. L. Porteus, "Investing in new parameter values in the discounted EOQ model," Naval Research Logistics Quarterly, vol. 33, no. 1, pp. 39-48, 1986. 


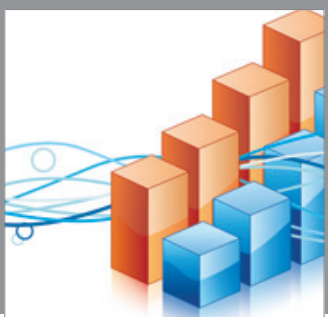

Advances in

Operations Research

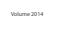

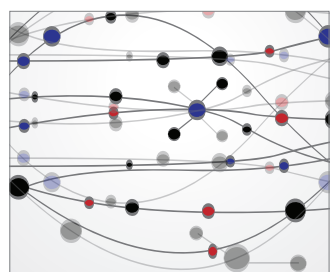

\section{The Scientific} World Journal
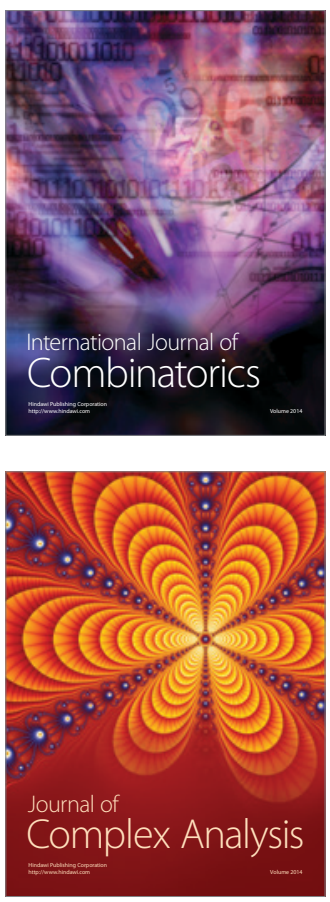

International Journal of

Mathematics and

Mathematical

Sciences
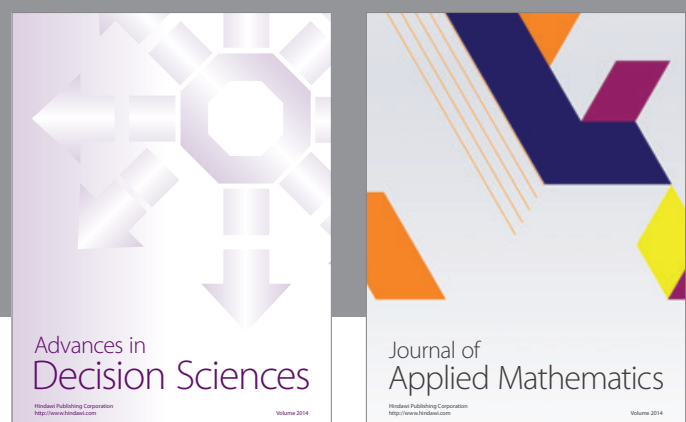

Journal of

Applied Mathematics
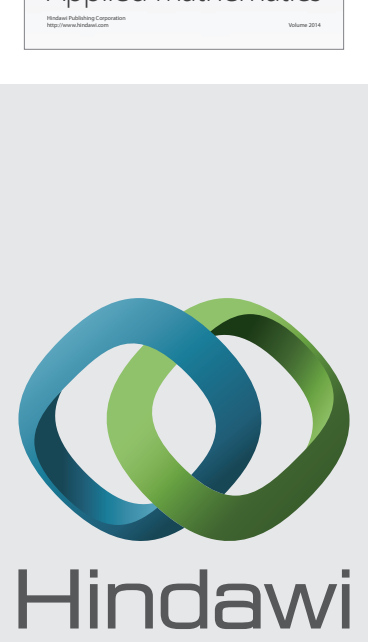

Submit your manuscripts at http://www.hindawi.com
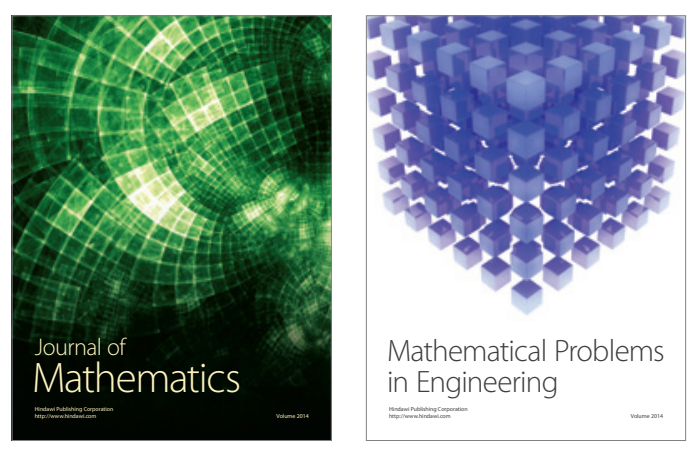

Mathematical Problems in Engineering
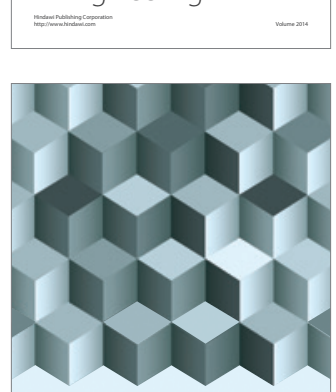

Journal of

Function Spaces
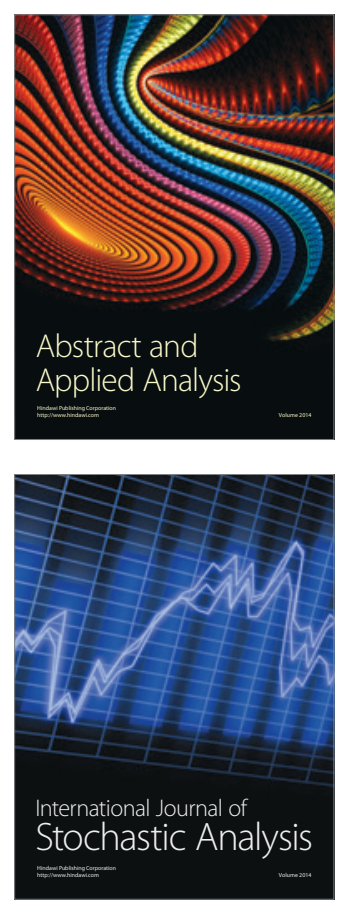

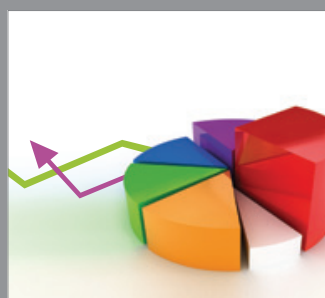

ournal of

Probability and Statistics

Promensencen
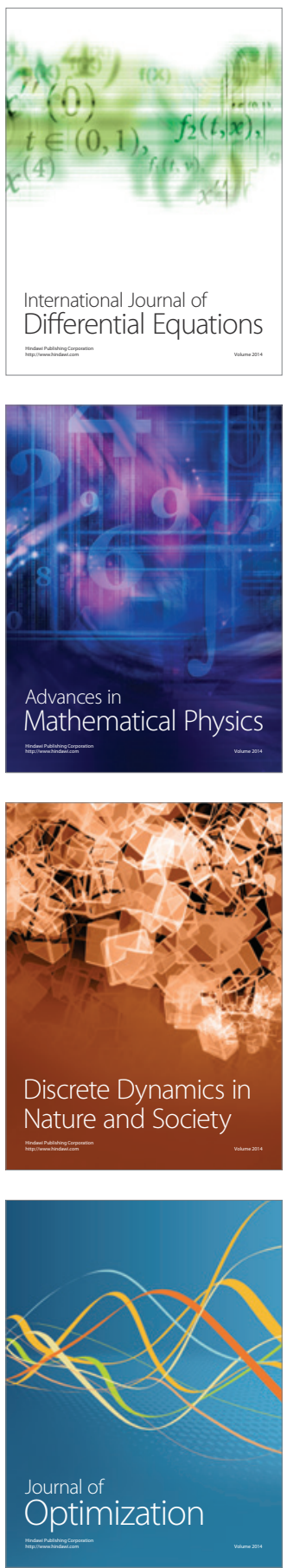